\title{
Improvement of exercise capacity following neonatal respiratory failure: A randomized controlled trial
}

\author{
Leontien C. C. Toussaint-Duyster ${ }^{1,2}$ \\ Tim Takken $^{3}$ | Wouter J. Harmsen ${ }^{1}$ \\ Monique H. M. van der Cammen-van Zijp ${ }^{1,2}$ \\ Ivo de Blaauw $^{1}$ | Rene M. H. Wijnen ${ }^{1}$ | Joost van Rosmalen ${ }^{5}$ | Hanneke IJsselstijn ${ }^{1}$
}

\begin{abstract}
${ }^{1}$ Intensive Care and Department of Pediatric Surgery, Erasmus Medical Center-Sophia Children's Hospital, Rotterdam, The Netherlands

${ }^{2}$ Section of Physical Therapy, Department of Orthopedics, Erasmus Medical Center-

Sophia Children's Hospital, Rotterdam, The Netherlands

${ }^{3}$ Child Development and Exercise Center, Wilhelmina Children's Hospital University Medical Center, Utrecht, The Netherlands

${ }^{4}$ Department of Pediatrics, Radboud University Medical Center-Amalia Children's Hospital, Nijmegen, The Netherlands

${ }^{5}$ Department of Biostatistics, Erasmus Medical Center, Rotterdam, The Netherlands
\end{abstract}

\section{Correspondence}

Leontien C. C. Toussaint-Duyster, Pediatric Physical Therapy, Erasmus MC—Sophia Children's Hospital, SK-0327, Wytemaweg 80, 3015 CN Rotterdam, The Netherlands. Email: 1.toussaint@erasmusmc.nl

\section{Funding information}

Stichting Rotterdams Kinderrevalidatie Fonds Adriaanstichting, Grant/Award Number: 2011/0128
Exercise capacity deteriorates in school-aged children born with major anatomical foregut anomalies and/or treated with extracorporeal membrane oxygenation. The aim of the present study was to evaluate whether exercise capacity can be improved in the short term and long term in children born with anatomical foregut anomalies and/or treated with extracorporeal membrane oxygenation. Therefore, we evaluated two different interventions in this single-blinded randomized controlled trial. Forty participants were randomly assigned to group A: standardized anaerobic high-intensity interval training plus online lifestyle coaching program, B: online lifestyle coaching program only, or C: standard of care. Inclusion criteria were as follows: score $\leq-1$ standard deviation (SD) on the Bruce protocol. Exercise capacity was assessed at baseline (T0), after 3 months (T1), and after 12 months (T2). Exercise capacity improved over time: mean (SD) standard deviation score (SDS) endurance time: T0 -1.91 (0.73); T1 -1.35 (0.94); T2 -1.20 (1.03): both $P<.001$. No significant differences in maximal endurance time were found at T1 (group A-C: estimated mean difference (SDS): $0.06 P=.802$; group B-C: $-0.17 P=.733$ ) or T2 (group A-C: $-0.13 P=.635$; group B-C: $-0.18 P=.587$ ). Exercise capacity improved significantly over time, irrespective of the study arm. Not only residual morbidities may be responsible for reduced exercise capacity. Parental awareness of reduced exercise capacity rather than specific interventions may have contributed. Monitoring of exercise tolerance and providing counseling on lifestyle factors that improve physical activity should be part of routine care, and aftercare should be offered on an individual basis.

\section{K E Y W O R D S}

clinical trial, exercise capacity, family factors, major anatomical foregut anomalies, neonatal extracorporeal membrane oxygenation, online coaching, physical activity, training program

\section{1 | INTRODUCTION}

Children born with major anatomical foregut anomalies, for example, congenital diaphragmatic hernia (CDH), esophageal 
(CAA) may require ECMO, for example, meconium aspiration syndrome or sepsis. ${ }^{1,2}$

We previously showed that children with $\mathrm{CDH}$ and EA are at risk for long-term persistent respiratory morbidity, ${ }^{2,3}$ reduced exercise capacity, ${ }^{4}$ and motor function problems. ${ }^{5}$ Moreover, exercise capacity in neonatal ECMO survivors deteriorated between 5 and 12 years of age. ${ }^{6}$ Reduced exercise capacity can lead to inactivity ${ }^{7}$ and, in turn, to gross motor function problems. It may also lead to less participation in daily life activities, and consequently a greater risk for secondary disease. $^{8}$

In general, early intervention might improve children's exercise capacity and therewith motor performance. In children with neonatal respiratory failure, intervention programs to improve exercise capacity are lacking. The question is whether persistent respiratory morbidity hampers improvement of exercise capacity. Training programs in children born with cardiac anomalies proved to be beneficial in increasing exercise capacity. ${ }^{9,10}$ The few studies available on online coaching programs aiming to promote physical activity in children and in adults suggested that these programs may be beneficial. ${ }^{11,12}$ We hypothesized that a short training program might be insufficient to achieve sustained improvement of exercise capacity in children with CAA and/or neonatal ECMO but that coaching aiming at influencing every day activities would render better effect.

We therefore conducted a randomized controlled trial (RCT) to answer the following questions.

\section{1 | Primary research question}

- Can exercise capacity be improved in these children?

\section{2 | Secondary research questions}

- Does intervention with either a standardized training program plus online lifestyle coaching or online lifestyle coaching only improve exercise capacity in the short term (3 months) and long term (12 months) and will intervention render greater effect than the standard of care?

- Does intervention with either a standardized training program plus online lifestyle coaching or online lifestyle coaching only improve motor performance, daily life activity, quality of life, and/or perceived motor competence (PMC)?

- Do family factors such as parental health status and their proactive coping influence the change in exercise capacity?

- Is change in exercise capacity diagnosis-dependent?

\section{2 | PATIENTS AND METHODS}

\section{1 | Participants}

From January 2013 till October 2015, subjects were recruited from the interdisciplinary follow-up program in our hospital. ${ }^{4,6}$ Children fulfilling the inclusion criteria received written information after routine assessments at age 8 or 12 years. Eligible children not yet scheduled were contacted by mail. Inclusion criteria were as follows: age 7-12 years; diagnosis of $\mathrm{CDH}, \mathrm{EA}, \mathrm{CPAM}$, and/or neonatal ECMO; and a score of at least 1 standard deviation (SD) below the norm on the maximal cardiopulmonary exercise test (CPET; Bruce protocol). ${ }^{13}$ Exclusion criteria were as follows: delayed motor function (ie, percentile score $<6$ on Movement Assessment Battery for Children second edition [MABC2]) requiring intervention by a pediatric physical therapist (PPT); inability or contraindication to perform CPET; and insufficient command of Dutch language (child or parents). From March 2014 onwards, potentially eligible children treated in Radboud University MC-Amalia Children's Hospital (Nijmegen, the Netherlands) were recruited by mail as well.

Ethical approval was granted by the institutional review board (MEC-2011-475), and all parents and children up from 12 years provided written informed consent. Clinical Trial register: Netherlands Trial Registry: NTR3729.

\subsection{Design}

In this RCT, participants were randomly assigned to a standardized training program for the child plus online lifestyle coaching for child and family (intervention group A), online lifestyle coaching as in group A (intervention group B), or standard of care (non-intervention group C). A note must be made about the standard of care. Our standardized follow-up program consists of assessments at the ages of 6,12 , and 24 months, and 5, 8, 12, 17 years, which implies that-at school age-the follow-up assessments take place at several years' intervals. In this $\mathrm{RCT}$, the children in the non-intervention group received standard of care, but were invited for extra follow-up assessments 3 and 12 months after the baseline assessment $(T=0)$. Outcome measures were assessed at baseline (T0), after 3 months (T1), and after 12 months (T2) (Supporting Information). Assessments were performed by an experienced assessor blinded to group assignment. Outcomes were also analyzed blinded to group assignment. The setting was the outpatient clinic of our level III university hospital (Erasmus MC). 


\section{3 | Sample size calculation}

Data of a previous study performed in school-aged neonatal ECMO survivors were used for the sample size calculation. ${ }^{6}$ We hypothesized that improvement of the outcome of the Bruce protocol at T1 would be 1 standard deviation score (SDS) greater for participants in group A (compared with C) and 0.9 SDS greater for participants in group B (compared with C). We calculated that a sample size of 33 subjects per study arm would be required to obtain a power of $88 \%$ and $80 \%$ for groups A and B, respectively, with a twosided significance level of 0.025 to adjust for the effects of multiple testing. We thus aimed to include 99 participants.

\subsection{Randomization}

We used a random number generator to generate randomly ordered numbers from 1 to 99 . A priori, it was established that numbers 1-33 would be allocated to group A, numbers 34-66 to group B, and numbers 67-99 to group C. These randomly ordered numbers were put into sequentially numbered (ie, 1, 2, 3 to 99), opaque, sealed envelopes by an employee who was not involved in this study. The first included participant received envelope 1, the second participant envelope 2 , and so on.

\section{5 | Intervention}

The interventions are described in more detail in Supporting Information. This file includes a Table with detailed information on the anaerobic high-intensity interval training that was provided.

\subsection{1 | Group A: standardized training program for the child plus online lifestyle coaching for child and family}

The participants followed a standardized twice-weekly program of anaerobic high-intensity interval training for twelve consecutive weeks provided by a local community-based PPT. In the second half of the program, aerobic exercise training was extended. ${ }^{14}$

Besides, they took an online coaching program to increase physical activity, based on the Integrated Model for Change. ${ }^{15}$

\subsection{2 | Group B: online lifestyle coaching for child and family}

These participants only took the online coaching program as in group A.

\subsection{3 | Group C: standard of care}

Participants in the non-intervention group were routinely advised on doing physical activities and sports, supported with a hand-out.

\section{6 | Measurements}

All measurements are described in a short overview and in more detail in Supporting Information.

\subsection{1 | Baseline data}

The following baseline data were recorded: gender, age, diagnosis, gestational age, duration of artificial ventilation, and major cardiac anomalies. Furthermore, lung function ${ }^{16-18}$ and growth ${ }^{19}$ were assessed.

\subsubsection{Primary outcome}

\section{Exercise capacity}

Exercise capacity was measured on a motor-driven treadmill, according to the Bruce protocol. ${ }^{13}$

\subsubsection{Secondary outcome}

\section{Motor performance}

Motor performance was evaluated with the MABC-2. ${ }^{20}$

\subsection{4 | Children's self-reports}

Daily activity

Daily activity was evaluated with the daily activity questionnaire. $^{21}$

\section{Participation patterns, intensity, and preferences in} leisure and recreation activities

The Children's Assessment of Participation and Enjoyment (CAPE) was used to identify participation patterns (diversity scores) and the intensity of leisure and recreation activities (intensity scores) from children's own perspectives. Preferences were assessed with the Preferences for Activities of Children (PAC). ${ }^{22,23}$

\section{Quality of life}

Perceived quality of life was measured with the Pediatric Quality of Life Inventory (PedsQL) 4.0. ${ }^{24}$ 


\section{Perceived motor competence}

PMC and importance of motor competence (IMC) were measured with the motor self-perception questionnaire. ${ }^{25}$

\subsection{5 | Parental self-reports}

\section{Proactive coping competence}

The various competencies involved in proactive coping were assessed with the Utrecht Proactive Coping Competence Inventory (UPCC). ${ }^{26}$

\section{Health status}

Parental health status was assessed with the Short Form-36 which contains two summary measures: physical health component summary (PCS) and mental health component summary (MCS). ${ }^{27}$

\section{Online coaching program}

The online coaching program recorded the number of days participants logged into this program.

\section{7 $\quad$ Statistical analysis}

Differences in baseline characteristics between the study arms were evaluated with the Kruskal-Wallis test (continuous variables) and the chi-square test (categorical variables). The paired samples $t$ test was used to test whether exercise capacity changed over time (T1-T0 and T2-T0). To test whether the secondary outcomes changed over time (T1-T0 and T2-T0), we used paired samples $t$ tests or Wilcoxon signed-rank tests where appropriate. One-sample $t$ tests were used to evaluate whether the normally distributed data of lung function, exercise capacity, motor performance, and health status differed from population norms $(\mathrm{SDS}=0)$. We applied analysis of covariance (ANCOVA) to examine whether the study arm influenced continuous outcome variables with adjustment for the baseline measurements. This was also applied to examine whether the diagnosis influenced exercise capacity. A two-way interaction effect between the study arm and diagnosis group was added to the model if the interaction effect was statistically significant. ANCOVA was performed separately for T1 and T2. For categorical outcome variables, we used Fisher's exact tests to examine the effect of the study arm. We calculated the Pearson correlation between the change in exercise capacity and the change in motor performance or the change in number of days that participants walk or ride a bike for school-home transfers. Besides, we calculated the Pearson correlation between the change in exercise capacity and the proactive coping competence or health status of the parents. The Mann-Whitney test served to examine whether there is a difference between the intervention groups in number of days the participants logged into the online coaching program. There were some missing values in the outcomes of the statistical analyses, which were handled by performing complete case analyses. No missing values were obtained in the independent variables.

The analyses were performed with SPSS version 24. The level of significance was set to 0.05 , but Bonferroni correction was applied in the ANCOVA models to adjust for multiple comparisons, resulting in an adjusted significance level of 0.025 .

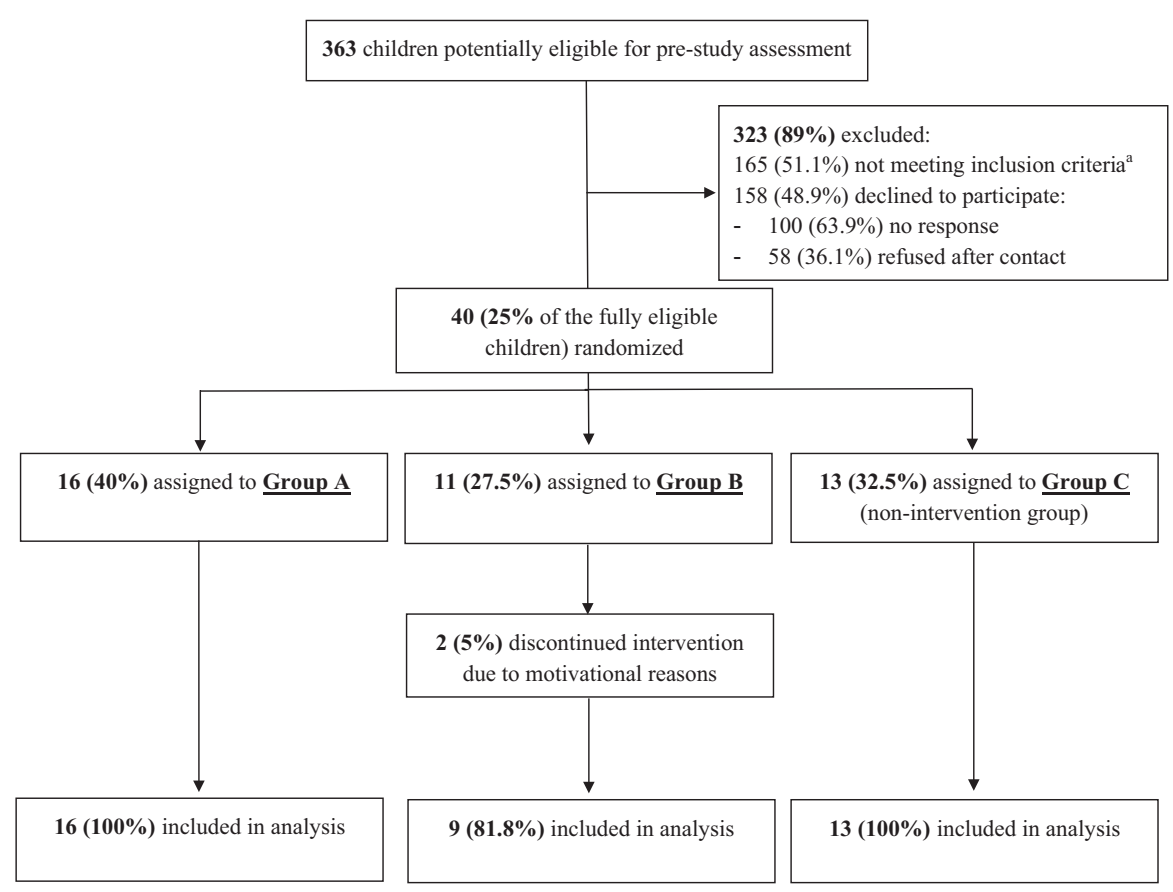

F IG U RE 1 Inclusion flow diagram. ${ }^{\mathrm{a}} 127(77.0 \%)$ children had a normal exercise capacity (SDS endurance time $>-1$ on the Bruce protocol); 9 (5.5\%) children had (severely) delayed motor function (percentile score $<6$ at MABC-2) requiring intervention by an PPT; 29 (17.5\%) children had inability or medical contraindication to perform the maximal cardiopulmonary exercise test. 


\section{3 | RESULTS}

\section{1 | Participants}

We considered 363 children potentially eligible: 158 (48.9\%) did not respond or refused participation after initial contact; $165(51.1 \%)$ were evaluated but not included (Figure 1), mostly because the exercise capacity score was normal (in $77 \%$ ). Eventually, 40 children participated (25\% of the fully eligible children) and were randomly assigned to one of the three study arms. Only 2 (5\%) participants, in group B, discontinued the intervention (Figure 1).

The PMC and IMC were filled in by all 40 participants at assessments; the other questionnaires by $38 / 40$ at T0, 35/39 at $\mathrm{T} 1$, and $35 / 38$ at $\mathrm{T} 2$.

Relevant baseline characteristics are listed in Table 1. Spirometry parameters were significantly below normal (all $P \leq .001$ ). Bronchodilation was not provided in nine participants with tracheomalacia or previous clinical deterioration after bronchodilation. Five participants (12.5\%) had reversible airflow obstruction; the median (interquartile range [IQR]) relative change in forced expiratory volume in 1 second was 14 (13-16). The baseline characteristics between participants in the different study arms did not differ significantly (Table 1).

\section{2 | Primary outcome}

\subsection{1 | Exercise capacity}

Participants performed maximally on the exercise test at all assessments. Mean SDS (SD) endurance time improved significantly over time: T0 $-1.91(0.73)$; $\mathrm{T} 1-1.35(0.94)$; T2 -1.20 (1.03): T0-T1 and T0-T2 $P<.001$. It was significantly below normal at all measurement points: all $P<.001$ and also for all study arms: $P<.05$ (Table 2 ).

TA B L E 1 Baseline characteristics

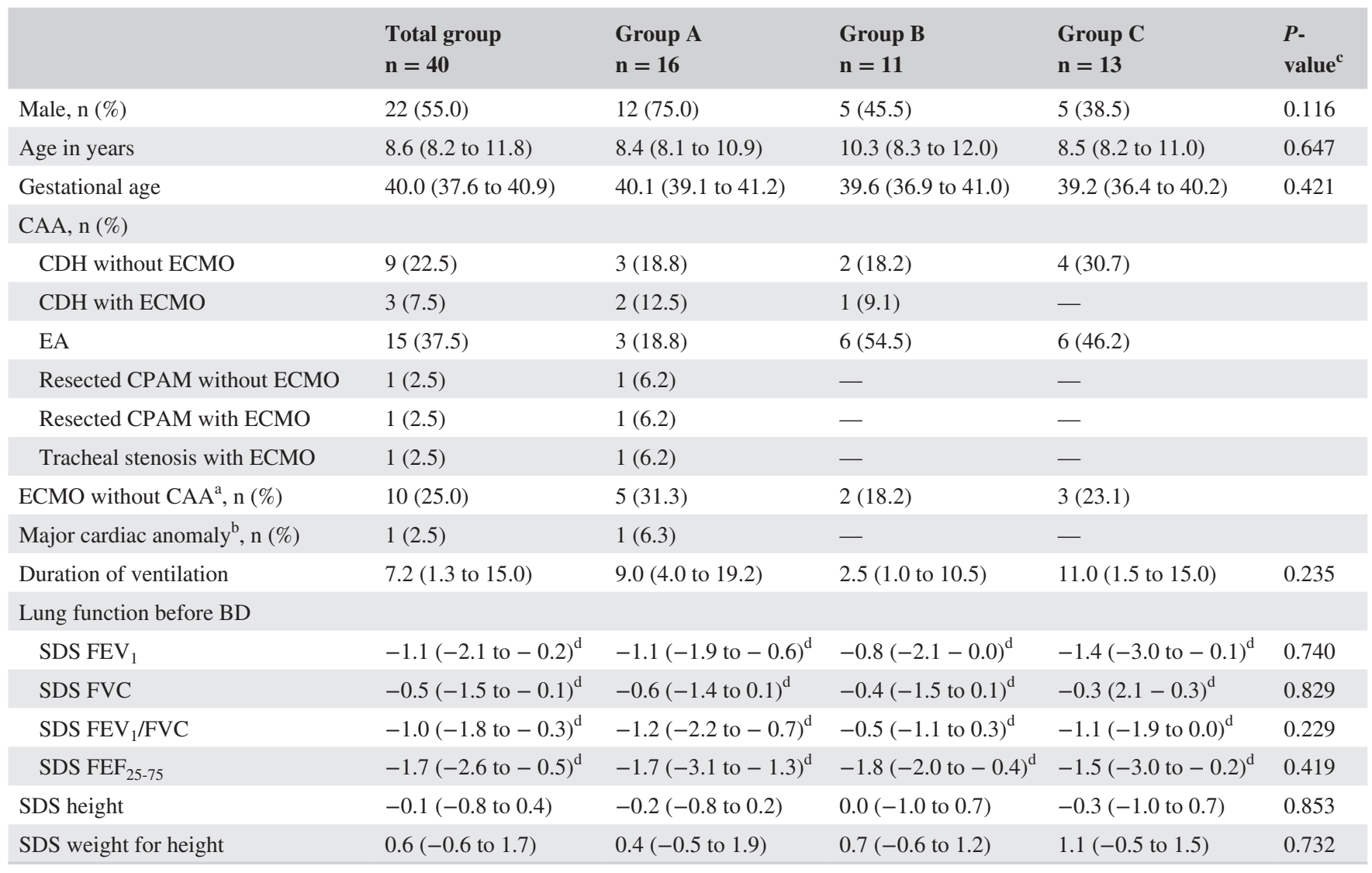

Note: Data presented as median (interquartile range), unless otherwise stated. Group A = standardized training program for the child plus online lifestyle coaching for the child and its family; group $\mathrm{B}=$ online lifestyle coaching for the child and its family; group $\mathrm{C}=$ standard of care.

Abbreviations: $\mathrm{BD}$, bronchodilation; $\mathrm{CAA}$, congenital anatomical anomalies; $\mathrm{CDH}$, congenital diaphragmatic hernia; EA, esophageal atresia; CPAM, congenital pulmonary airway malformation; ECMO, extracorporeal membrane oxygenation; $\mathrm{FEV}_{1}$, forced expiratory volume in 1 second; $\mathrm{FEF}_{25-75}$, forced expiratory flows between $25 \%$ and $75 \%$ of vital capacity; FVC, forced vital capacity; n, number of patients; SDS, standard deviation score.

${ }^{\mathrm{a}} \mathrm{ECMO}$ without CAA: meconium aspiration syndrome $\mathrm{n}=6$, persistent pulmonary hypertension of the newborn $\mathrm{n}=2$, sepsis $\mathrm{n}=1$, respiratory syncytial virus $\mathrm{n}=1$.

${ }^{b}$ Vitium cordis: double outlet right ventricle, open ductus Botalli with left-right shunt and atrial septal defect with surgical correction.

${ }^{\mathrm{c}}$ Kruskal-Wallis test or chi-square test: difference between study arms.

${ }^{\mathrm{d}}$ One-sample $t$ test: significantly below normal: $P \leq .001$. 
No significant differences in change in endurance time were found between the study arms (Table 3 and Figure 2). Table $\mathrm{S} 3$ shows the change in exercise capacity in the individual participants per study arm. The majority improved over time.

\subsection{2 | Exercise capacity per diagnosis group: CAA vs neonatal ECMO without CAA}

In participants with CAA $(\mathrm{n}=30)$, exercise capacity improved over time: T0 -2.00 (0.79); T1 $-1.44(0.99)$; T2 -1.21 (1.11); T0-T1 and T0-T2 $P<.001$. The same was true for the neonatal ECMO-treated participants without CAA $(\mathrm{n}=10)$ : T0 -1.64 (0.48); T1 -1.11 (0.79); T2 -1.18 (0.82); T0-T1 $P=.029$ and T0-T2 $P=.030$. No significant differences in endurance time were found between the diagnosis groups at T1 (estimated mean difference SDS (95\% CI): $0.18(-0.42-0.66) P=.660)$ or T2 $(0.37(-0.16-0.91)$ $P=.164)$. There was no significant interaction effect between the diagnosis and the study arm at $\mathrm{T} 1(P=.286)$ or $\mathrm{T} 2(P=.305)$.

\section{3 | Secondary outcome}

\subsection{1 | Motor performance}

Motor performance improved over time: mean (SD) SDS Total Impairment Score (TIS): T0 -0.25 (0.95); T2 0.14 (0.96): $P=.002$. The mean SDS TIS at T0 and T2 did not significantly differ from that in the reference population (see Table S4). We found no significant differences in motor performance between the study arms (see Table S5).

No significant correlation was found between the change (T0-T2) in mean SDS endurance time and change (T0-T2) in mean SDS TIS $(r=.104, P=.535)$.

\subsection{2 | Questionnaires}

\section{Self-reports}

Daily activity. Scores on the daily activity questionnaire did not significantly change over time: T0-T1 $P=.714$; T0-T2 $P=.765$ (see Table S4). Still, at T1 and T2 more participants spent an average/or above-average amount of time (>1 h/wk) on sports (T0 44.8\%, T1 $57.2 \%$ and T2 $65.7 \%)$. At the same time, more participants spent aboveaverage time $(>3.5 \mathrm{~h} / \mathrm{wk})$ on watching TV or playing video games (T0 23.7\%, T1 34.4\% and T2 34.4\%). The scores were not significantly different between the study arms at all measurement points: T0 $P=.523$; T1 $P=.376$; T2 $P=.481$ (data not shown).
The change in number of days that participants walk or ride a bike for school-home transfers was significantly correlated with the change in endurance time $(r=.375$, $P=.045)$.

Participation and preferences. The total diversity, total intensity, and total preferences scores had not significantly changed at $\mathrm{T} 1$ from $\mathrm{T} 0$, but had decreased significantly at T2 (see Table S4). The CAPE and the PAC results did not significantly differ between the study arms (see Table S5).

Quality of life. The scores of the physical, social, and total functioning scale increased significantly over time (see Table S4). No significant differences were found between the study arms (see Table S5).

Perceived motor competence. PMC did not significantly change over time: T0-T1 $P=.488$; T0-T2 $P=.431$ (Table S4). The study arm had no significant effect on PMC (see Table S5).

Also, IMC did not significantly change over time: T0-T1 $P=.475 ;$ T0-T2 $P=.299$ (Table S4) and the study arm had no significant effect (see Table S5).

\section{Parental self-reports}

Proactive coping competence. The raw score (SD) on the UPCC was $2.97(0.47)$ at T0, which indicates that parents considered themselves competent in proactive coping. Parental proactive coping did not influence the change in exercise capacity as this score did not significantly correlate with the change in mean SDS endurance time $(r=.044$, $P=.799$ ).

Scores on the UPCC did not significantly change over time (data not shown). No significant differences in the UPCC score were found between the study arms (see Table S5).

Health status. The mean (SD) SDS on the PCS was 0.40 (0.66) and on the MCS 0.08 (0.71) at T0. The mean SDS PCS was significantly higher than in the reference population at all measurement points, and the mean (SD) SDS MCS was normal at all points (data not shown). Parental health status did not influence the change in exercise capacity as parental health status and the change in mean SDS endurance time of the participants were not significantly correlated (PCS: $r=-.095, P=.580$; MCS: $r=.103, P=.551)$.

Parental health status did not change significantly over time (data not shown). No significant differences were found between the study arms on the scores of the PCS and MCS (see Table S5).

Online coaching program. No significant difference was found between groups A and B in the number of days 
TA B L E 2 Primary outcome: SDS endurance time over time

\begin{tabular}{lllll} 
& Total group & Group A & Group B & Group C \\
T0 & $\mathrm{n}=40$ & $\mathrm{n}=16$ & $\mathrm{n}=11$ & $\mathrm{n}=13$ \\
Mean (SD) SDS & $-1.91(0.73)^{\mathrm{a}}$ & $-1.84(0.69)^{\mathrm{a}}$ & $-1.63(0.46)^{\mathrm{a}}$ & $-2.23(0.89)^{\mathrm{a}}$ \\
T1 & $\mathrm{n}=39$ & $\mathrm{n}=16$ & $\mathrm{n}=10$ & $\mathrm{n}=13$ \\
Mean (SD) SDS & $-1.35(0.94)^{\mathrm{a}}$ & $-1.23(0.95)^{\mathrm{a}}$ & $-1.16(1.06)^{\mathrm{b}}$ & $-1.66(0.84)^{\mathrm{a}}$ \\
T2 & $\mathrm{n}=38$ & $\mathrm{n}=16$ & $\mathrm{n}=9$ & $\mathrm{n}=13$ \\
Mean (SD) SDS & $-1.20(1.03)^{\mathrm{a}}$ & $-1.17(1.14)^{\mathrm{a}}$ & $-0.88(1.17)$ & $-1.45(0.77)^{\mathrm{a}}$ \\
\hline
\end{tabular}

Note: T0 baseline assessment; T1 assessment after 3 mo; T2 assessment after 12 mo. Group A = standardized training program for the child plus online lifestyle coaching for the child and its family; group $\mathrm{B}=$ online lifestyle coaching for the child and its family; group $\mathrm{C}=$ standard of care.

Abbreviations: SD, standard deviation; SDS, standard deviation score.

${ }^{\text {a }}$ significantly below the norm (compared with SDS $=0$ ): $P \leq .001$ one-sample $t$ test.

${ }^{\mathrm{b}}$ significantly below the norm (compared with SDS $=0$ ): $P<.05$ one-sample $t$ test.

\begin{tabular}{lll} 
& Group A vs Group C & $\begin{array}{l}\text { Group B vs } \\
\text { Group C }\end{array}$ \\
T1-T0 & $0.06(-0.45$ to 0.58$)$ & $-0.10(-0.70$ to \\
Estimated mean difference SDS $(95 \% \mathrm{CI})^{\mathrm{a}}$ & $P=.802$ & $0.50)$ \\
& & $P=.733$ \\
T2-T0 & $-0.13(-0.68$ to 0.42$)$ & $-0.18(-0.84$ to \\
Estimated mean difference SDS $(95 \% \mathrm{CI})^{\mathrm{a}}$ & $P=.635$ & $0.48)$ \\
& & $P=.587$ \\
\hline
\end{tabular}

Note: T0 baseline assessment; T1 assessment after 3 mo; T2 assessment after 12 mo. Group A = standardized training program for the child plus online lifestyle coaching for the child and its family; Group B = online lifestyle coaching for the child and its family; Group $\mathrm{C}=$ standard of care.

Abbreviations: CI, confidence interval; SD, standard deviation; SDS, standard deviation score.

${ }^{\mathrm{a}}$ Based on an analysis of covariance model adjusted for baseline measurement.

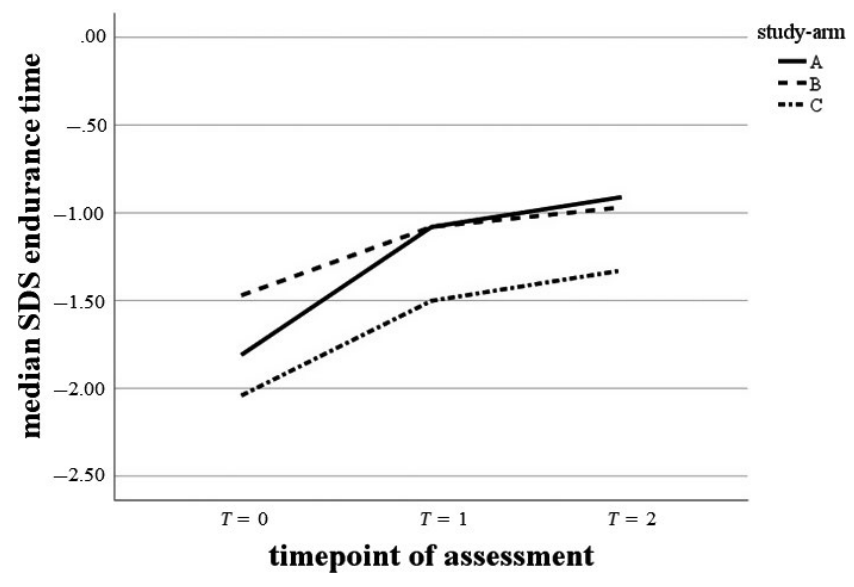

F I G URE 2 Median SDS endurance time over time of the study arms. Study arm A = standardized training program for the child plus online lifestyle coaching for the child and its family; study $\operatorname{arm} \mathrm{B}=$ online lifestyle coaching for the child and its family; study arm $\mathrm{C}=$ standard of care. T0 baseline assessment; T1 assessment after 3 mo; T2 assessment after 12 mo. Abbreviation: SDS, standard deviation score

participants logged into the online coaching program (group A: median (IQR) number of days: 20 (10-45); B: 14 (9-24), $P=.256)$.
Inclusion stop. The calculated sample size was not achieved (Figure 1). Active nationwide recruitment of participants with extension of the inclusion period had insufficient effect. From March 2014 onwards, potentially eligible children treated in Radboud University MC-Amalia Children's Hospital (Nijmegen, the Netherlands) were invited as well. Almost half of the patients and their parents (43/98 44\%) responded, and thirty of them $(31 \%)$ provided written informed consent. After this nationwide recruitment, twelve of the thirty patients (40\%) who had provided written informed consent met the inclusion criterion of a score of at least 1 standard deviation (SD) below the norm on the maximal CPET and participated in the trial. As we did not expect a higher inclusion rate within the next years, the principal investigator stopped the inclusion after almost 3 years, in October 2015. This decision to stop was not influenced by preliminary results as we analyzed our data after the last included participant had finished the program.

\section{4 | DISCUSSION}

In this RCT, we showed that impaired exercise capacity improved in children with persistent respiratory morbidity following neonatal surgery for CAA and/or neonatal 
ECMO. Improvement was not only seen in the intervention groups, but also occurred in the non-intervention group. Motor performance and self-reported physical and social functioning improved also in all three study arms.

The parents of our participants reported normal health status and normal competence in proactive coping. Their well-developed proactive coping competence may have contributed to change from a semi-active to an active lifestyle, including physically active school-home transfers, and hence to the improvement of exercise capacity in participants of all study arms.

Studies in children with other chronic conditions, such as cardiac anomalies, also found that training programs increased exercise tolerance. ${ }^{9,10,28}$ However, these studies did not include controls. RCTs on improvement of exercise capacity in children are scarce: contradictory results have been reported for children with acute lymphatic leukemia. ${ }^{29,30}$ Comparison with the present study is difficult due to differences in study population, intervention type, and primary outcome parameter. Interval training has been proven effective in ambulatory children with spina bifida. ${ }^{31}$

The CAPE scores in our study were in concordance with those of healthy Dutch children. ${ }^{23,32}$ Therefore, it can be argued whether participation in our population would improve. Moreover, this questionnaire was developed in rehabilitation settings and might be more suitable for children with more severe physical disabilities, such as cerebral palsy.

Strengths of our study are the randomized controlled study design with only two dropouts from online coaching. The wide range in the use of the online coaching program suggests that not everyone adhered to this intervention. Future interventions should probably focus on a peer support system. The lack of peer support system use is a commonly reported problem in online interventions. ${ }^{33}$

The question is whether the lack of a positive effect of the interventions can be explained by study limitations. For one, the calculated sample size was not achieved despite nationwide recruitment, so that the group sample sizes were relatively small. Despite the low recruitment rate, the primary outcome exercise capacity improved significantly over time. The low recruitment rate increased the probability of not finding significant differences between the study arms. Besides, it is assumable that participation in this study created awareness of reduced exercise capacity and therewith lifestyle changes by the participants and their parents of the non-intervention group, a phenomenon known as the Hawthorne effect. ${ }^{34}$ Also, the follow-up assessments after 3 and 12 months, which in our regular follow-up program are scheduled only after 4 to 5 years, probably contributed to this phenomenon. The positive significant correlation between the change in number of days that participants walk or ride a bike for school-home transfers and the change in endurance time, also in the non-intervention group, supports this effect. Secondly, the used questionnaires may not have been sensitive enough to evaluate changes in daily activities, especially if relatively young children have to recall events in the past week (daily activity questionnaire) or even in the past 4 months (CAPE). Moreover, children tend to overrate physical activities. ${ }^{35}$ Therefore, our data do not allow concluding what factors contributed to improvement of exercise capacity. Thirdly, we refrained from using an activity tracker to measure daily activities. At the start of this study, the available equipment was not suitable to reliably record other physical activities than walking and running in children, as cycling and roller skating. ${ }^{36}$ For future research, the use of activity trackers should be considered. Fourthly, it can be debated whether the interventions were optimal for our population. High-intensity training has been shown to be beneficial in RCTs with healthy or obese children, ${ }^{37,38}$ and corresponds with the intensity of physical activities in the everyday life of school-aged children. As the use of the online coaching program was subject to variability, and we only had dropouts in group B, we assume that online individual coaching will only be beneficial for selected families.

\section{5 | PERSPECTIVE}

In previous studies, we showed that children born with major anatomical foregut anomalies and/or treated with ECMO are at risk for long-term respiratory morbidity, ${ }^{2,3}$ reduced exercise capacity, ${ }^{4}$ and even deterioration of exercise capacity. ${ }^{6,39}$ In this RCT, we aimed to evaluate whether these children's exercise capacity can be improved. We showed that exercise capacity improved significantly over time, irrespective of the intervention. This implies that residual morbidities are not the only factor responsible for reduced exercise capacity. Enhanced awareness of impaired exercise tolerance might have resulted in improvement over time in all three study arms. Parental proactive coping competence can stimulate a more physically active lifestyle in their child. Our observations have implications for the counseling of children and their parents. We speculate that parents of children who survived neonatal critical illness consider their child more vulnerable than the parents of healthy children and may therefore be reluctant to encourage physical activities in childhood. ${ }^{40}$ Close monitoring and counseling from an early stage onwards could improve physical activity and should be part of routine care.

\section{ACKNOWLEDGEMENTS}

The authors thank HJG van den Berg-Emons for advice, E. Zwarter for support to implement the online coaching program, and K. Hagoort for editorial advice. Especially, we thank the children and their families for participating in this study. 


\section{REFERENCES}

1. ECLS Registry Report. International Summary. Ann Harbor, MI: Extracorporeal Life Support Organization; 2017.

2. Spoel M, Laas R, Gischler SJ, et al. Diagnosis-related deterioration of lung function after extracorporeal membrane oxygenation. Eur Respir J. 2012;40:1531-1537.

3. Spoel M, Marshall H, IJsselstijn H, et al. Pulmonary ventilation and micro-structural findings in congenital diaphragmatic hernia. Pediatr Pulmonol. 2016;51:517-524.

4. van der Cammen-van Zijp MH, Gischler SJ, Mazer P, van Dijk M, Tibboel D, IJsselstijn H. Motor-function and exercise capacity in children with major anatomical congenital anomalies: an evaluation at 5 years of age. Early Hum Dev. 2010;86:523-528.

5. van der Cammen-van Zijp MHM, Janssen A, Raets MM, et al. Motor performance after neonatal extracorporeal membrane oxygenation: a longitudinal evaluation. Pediatrics. 2014;134:e427-435.

6. van der Cammen-van Zijp MHM, Gischler SJ, Hop WCJ, et al. Deterioration of exercise capacity after neonatal extracorporeal membrane oxygenation. Eur Respir J. 2011;38:1098-1104.

7. Durstine JL, Painter P, Franklin BA, Morgan D, Pitetti KH, Roberts SO. Physical activity for the chronically ill and disabled. Sports Med. 2000;30:207-219.

8. Boreham C, Riddoch C. The physical activity, fitness and health of children. J Sports Sci. 2001;19:915-929.

9. Jacobsen RM, Ginde S, Mussatto K, Neubauer J, Earing M, Danduran M. Can a home-based cardiac physical activity program improve the physical function quality of life in children with Fontan circulation? Congenit Heart Dis. 2016;11:175-182.

10. Zöller D, Siaplaouras J, Apitz A, et al. Home exercise training in children and adolescents with pulmonary arterial hypertension: a pilot study. Pediatr Cardiol. 2017;38:191-198.

11. McPherson AC, McAdam L, Keenan S, et al. A feasibility study using solution-focused coaching for health promotion in children and young people with Duchenne muscular dystrophy. Dev Neurorehabil. 2017;21:121-130.

12. Stut W, Deighan C, Armitage W, Clark M, Cleland JG, Jaarsma T. Design and usage of the heartcycle education and coaching program for patients with heart failure. JMIR Res Protoc. 2014;3:e72.

13. van der Cammen-van Zijp MHM, van den Berg-Emons RJG, Willemsen SP, et al. Exercise capacity in Dutch children: new reference values for the Bruce treadmill protocol. Scand J Med Sci Sports. 2010;20:e130-e136.

14. Takken T. Inspanningsfysiologie bij kinderen. Houten, The Netherlands: Bohn Stafleu van Loghum; 2008.

15. de Vries H, Mudde A, Leijs I, et al. The European Smoking Prevention Framework Approach (EFSA): an example of integral prevention. Health Educ Res. 2003;18:611-626.

16. Miller MR, Hankinson J, Brusasco V, et al. Standardisation of spirometry. Eur Respir J. 2005;26:319-338.

17. Quanjer PH, Stanojevic S, Cole TJ, et al. Multi-ethnic reference values for spirometry for the 3-95-yr age range: the global lung function 2012 equations. Eur Respir J. 2012;40:1324-1343.

18. Casan P, Roca J, Sanchis J. Spirometric response to a bronchodilator-Reference values for healthy-children and adolescents. B Eur Physiopath Res. 1983;19:567-569.

19. Schönbeck Y, Talma H, van Dommelen P, et al. The world's tallest nation has stopped growing taller: the height of Dutch children from 1955 to 2009. Pediatr Res. 2013;73:371-377.
20. Smits-Engelsman B. Manual Movement Assessment Battery for Children, 2nd edn. Amsterdam, Netherlands: Pearson; 2010.

21. Ooijendijk W, Wendel-Vos W, de Vries S. Consensus Quenstionnaires Sports and Movement. Leiden, The Netherlands: TNO; 2007.

22. King G, Law M, King S, et al. Manual CAPE/PAC Children's Assessment of Participation and Enjoyment \& Preferences for Activities of Children. Amsterdam, The Netherlands: Pearson; 2004.

23. Bult MK, Verschuren O, Gorter JW, Jongmans MJ, Piskur B, Ketelaar M. Cross-cultural validation and psychometric evaluation of the Dutch language version of the Children's Assessment of Participation and Enjoyment (CAPE) in children with and without physical disabilities. Clin Rehabil. 2010;24:843-853.

24. Engelen V, Haentjens MM, Detmar SB, Koopman HM, Grootenhuis MA. Health related quality of life of Dutch children: psychometric properties of the PedsQL in the Netherlands. BMC Pediatr. 2009;9:68.

25. de Kloet A, Calame E, Reinders H, Smits-Engelsman BCM, Schoemaker M, Volman C. Vragenlijst gevoel van motorische competentie; "Hoe ik vind dat ik het doe?" Den Haag, The Netherlands: Pearson; 2005.

26. Thoolen BJ, de Ridder D, Bensing J, Gorter K, Rutten G. Beyond good intentions: the role of proactive coping in achieving sustained behavioural change in the context of diabetes management. Psychol Health. 2009;24:237-254.

27. Ware JE Jr. SF-36 health survey update. Spine. 2000;25:3130-3139.

28. Kotte EM, de Groot JF, Winkler AM, Huijgen BC, Takken T. Effects of the Fitkids exercise therapy program on health-related fitness, walking capacity, and health-related quality of life. Phys Ther. 2014;94:1306-1318.

29. Tanir MK, Kuguoglu S. Impact of exercise on lower activity levels in children with acute lymphoblastic leukemia: a randomized controlled trial from Turkey. Rehabil Nurs. 2013;38:48-59.

30. Marchese VG, Chiarello LA, Lange BJ. Effects of physical therapy intervention for children with acute lymphoblastic leukemia. Pediatr Blood Cancer. 2004;42:127-133.

31. de Groot JF, Takken T, van Brussel M, et al. Randomized controlled study of home-based treadmill training for ambulatory children with spina bifida. Neurorehabil Neural Repair. 2011;25:597-606.

32. Bult MK, Verschuren O, Lindeman E, Jongmans MJ, Ketelaar M. Do children participate in the activities they prefer? A comparison of children and youth with and without physical disabilities. Clin Rehabil. 2014;28:388-396.

33. Eysenbach G, Powell J, Englesakis M, Rizo C, Stern A. Health related virtual communities and electronic support groups: systematic review of the effects of online peer to peer interactions. $B M J$. 2004;328:1166.

34. McCambridge J, Witton J, Elbourne DR. Systematic review of the Hawthorne effect: new concepts are needed to study research participation effects. J Clin Epidemiol. 2014;67:267-277.

35. Chinapaw MJ, Mokkink LB, van Poppel MN, van Mechelen W, Terwee CB. Physical activity questionnaires for youth: a systematic review of measurement properties. Sports Med. 2010;40:539-563.

36. Robertson W, Stewart-Brown S, Wilcock E, Oldfield M, Thorogood M. Utility of Accelerometers to Measure Physical Activity in Children Attending an Obesity Treatment Intervention. $J$ Obes. 2011;2011:1-8. https://doi.org/10.1155/2011/398918. 
37. Baquet G, Gamelin FX, Mucci P, Thevenet D, Van Praagh E, Berthoin S. Continuous vs. interval aerobic training in 8- to 11-year-old children. J Strength Cond Res. 2010;24:1381-1388.

38. Lau PW, del Wong P, Ngo JK, Liang Y, Kim CG, Kim HS. Effects of high-intensity intermittent running exercise in overweight children. Eur J Sport Sci. 2015;15:182-190.

39. Toussaint-Duyster LCC, van der Cammen-van Zijp MHM, de Jongste JC, et al. Congenital diaphragmatic hernia and exercise capacity, a longitudinal evaluation. Pediatr Pulmonol. 2019;54:628-636.

40. Smith LJ, van Asperen PP, McKay KO, Selvadurai H, Fitzgerald DA. Reduced exercise capacity in children born very preterm. Pediatrics. 2008;122:e287-e293.

\section{SUPPORTING INFORMATION}

Additional supporting information may be found online in the Supporting Information section.

How to cite this article: Toussaint-Duyster LCC, van der Cammen-van Zijp MHM, Takken T, et al.

Improvement of exercise capacity following neonatal respiratory failure: A randomized controlled trial.

Scand J Med Sci Sports. 2019;00:1-10. https://doi. org/10.1111/sms.13604 